\title{
Chromatic perceptual learning
}

\author{
Paul T. Sowden ${ }^{1}$, Ian R. L. Davies ${ }^{1}$, Leslie A. Notman ${ }^{1}$, Iona \\ Alexander ${ }^{2}$ and Emre Özgen ${ }^{3}$ \\ ${ }^{1}$ Department of Psychology, University of Surrey, U.K., \\ ${ }^{2}$ Department of Experimental Psychology, University of Oxford, U.K. \\ and ${ }^{3}$ Department of Psychology, Bilkent University, Turkey.
}

\begin{abstract}
Perceptual learning has been shown on a wide variety of achromatic visual tasks. However, very little work has explored the possibility of improvements on chromatically based tasks. Here, we used a transfer of learning paradigm to assess the specificity of improvements at discriminating the orientation of a chromatically defined edge presented in luminance noise. Chromatic thresholds were estimated for two different hues and retinal locations, before and after a ten day training period. During training observers discriminated the orientation of a chromatic edge at just one location and hue. Whilst performance improved following training, these improvements failed to transfer across either retinal location or hue. Our findings suggest that improvements in chromatically-mediated discrimination may involve plasticity at early, retinotopically mapped, stages of visual analysis. Further, they suggest that categorical perception of colour might in part arise from chromatic perceptual learning at colour category boundaries.
\end{abstract}

\section{Introduction}

Perceptual learning involves an improvement in some perceptual judgement with practice. During the last twenty years there have been numerous demonstrations that the detection or discrimination of a range of visual attributes improves as a result of training (cf. Fahle \& Poggio 2002). For instance, the ability to judge simple attributes such as line orientation (Shiu \& Pashler 1992), vernier separation (Fahle \& Edelman 1993) and luminance contrast (Sowden, Rose \& Davies 2002) have all been shown to improve. These improvements are frequently found to be specific to dimensions of early visual analysis such as retinal position and stimulus orientation. Consequently, the inference is often made that the learning results from neural plasticity at the early stages of visual analysis that are selective for these dimensions of visual analysis. 
However, this inference has been challenged and it has been suggested that permanent changes to early visual analysis to enhance performance on a particular task would interfere with performance on other tasks that are reliant on the same early analysis. Instead, it has been argued that later stages learn to attend those outputs from early analyses that provide the optimal information for solving the task at hand (cf. Mollon \& Danilova 1996; Petrov, Dosher \& Lu 2005). Despite this argument, a variety of neuroimaging studies provide convergent evidence for an early locus of perceptual learning (e.g. Furmanski, Schluppeck \& Engel 2004; Pourtois, Rauss, Vuilleumier \& Schwartz 2008; Schwartz, Maquet \& Frith 2002).

A third view extends previous ideas by suggesting that later stages of analysis dynamically modify those earlier stages that optimize task performance whilst that task is being carried out (see Sowden \& Schyns 2006 for a review). In this way, changes are made only to as early a level of analysis as necessary to perform a given task and only on a temporary basis, thereby avoiding interference with the performance of other tasks reliant on those same stages of analysis.

Despite the wealth of studies showing perceptual learning, and the advances made in understanding its mechanisms, to our knowledge there has been almost no work conducted to explore the possibility that the chromatic perceptual system can exhibit learning in the same way as seen for many tasks performed using the achromatic system.

Consistent with the possibility of chromatic perceptual learning, evidence from categorical perception (CP) research suggests that our colour perception may change as a result of experience. Categorical perception refers to an enhanced ability to discriminate stimuli that fall either side of a category boundary compared to equally different stimuli that fall within a category. For instance, speakers of languages that differ in their colour vocabulary show differences in their performance on a variety of colour tasks that probe colour CP, and these differences are predicted by their respective colour vocabulary (Roberson, Davies \& Davidoff 2000). Further, category training results in the development of categorical perception effects at newly learned category boundaries, again consistent with the possibility that chromatic perception may change with experience (Özgen \& Davies 2002). Interestingly, recent work that explores whether $\mathrm{CP}$ is equivalent in the left and right visual fields finds that colour CP is most marked in the right visual field. As initial visual processing of the right visual field is carried out by the left hemisphere of the brain, where language centres typically reside, it has been suggested that colour CP results from an online dynamic influence of language on the visual analysis of colour (Drivonikou, Kay, Regier, Ivry, Gilbert, Franklin \& Davies 2007; Siok, Kay, Wang, Chan, Chen, Luke \& Tan 2009). Consistent with this is evidence showing that as children acquire their colour vocabulary so colour CP effects change from the left to the right visual field (Franklin, Drivonikou, Clifford, Kay, Regier \& Davies 2008). This suggestion, of an online influence of higher level language processing on earlier perceptual processing, is consistent with current ideas about the locus of perceptual learning effects. 
Consequently, in our experiments we sought to test directly whether chromatic perception can change as a result of training and to explore the likely neural locus of these changes. Specifically, we tested the specificity of chromatic perceptual learning to retinal position and hue.

\section{Method}

\subsection{Participants}

Fifteen observers (mean age 24; range: 19-31) were paid to participate. All had normal colour vision as assessed by the City University Colour Vision Test (Fletcher 1980).

\subsection{Apparatus and stimuli}

Participants sat $57 \mathrm{~cm}$ away from and at eye-level to a 21-inch Eizo Flexscan F980 CRT monitor (CIE, 1931, $\mathrm{x}, \mathrm{y}$ phosphor co-ordinates: $\mathrm{x}_{\text {red }}=0.614 \mathrm{y}_{\text {red }}=0.335 ; \mathrm{x}_{\text {green }}=0.277$ $\left.\mathrm{y}_{\text {green }}=0.599 ; \mathrm{x}_{\text {blue }}=0.155 \mathrm{y}_{\text {blue }}=0.072\right)$. Stimuli were generated by a Cambridge Research Systems (CRS; Rochester, UK) Visual Stimulus Generator (VSG) 2/3 graphics card. This uses a 12-bit per gun resolution palette-based graphics system to generate colour stimuli. The monitor and VSG system were calibrated using proprietary CRS software in combination with a CRS ColorCal colorimeter. Calibration involved measuring (in CIE 1931 Yxy co-ordinates) the response of the red, green and blue monitor guns in isolation and combination throughout their response range, thereby providing information on the monitor phosphor co-ordinates (in CIE 1931 xy co-ordinates as listed above), the dark point for each gun, and allowing calculation of the gamma function for each gun in isolation and their combination. This calibration information was used to define the monitor gamut (the region of colour space that the monitor can reproduce) and to ensure accurate and high resolution reproduction of the desired colours. The chromaticity and luminance co-ordinates (CIE, 1931, Y,X,y) of the resultant stimuli were then verified using the ColorCal colorimeter. Participants' responses were made with a game pad and they received auditory feedback.

Stimuli consisted of two semi-circles of colour abutted to make a circle of $10^{\circ} \mathrm{di}$ ameter displayed against a luminance matched background (mean luminance $30.5 \mathrm{~cd} /$ $\mathrm{m}^{2}$ ). The two halves of the circle were designed to be isoluminant. Nevertheless, due to display non-uniformity over space and time, and variation in the isoluminant point with retinal location (Schiller, Logothetis \& Charles 1991), it is possible that there may be very small residual luminance differences that could be used by observers to detect the chromatic boundary. To prevent this, on each trial, the stimuli were embedded in Gaussian luminance noise, which serves to swamp any residual luminance signal that could be used to detect the boundary (Snowden 2002). A fixation spot was always present in the centre of the screen. 
Training colours $=$ Munsell 7.5B and 7.5G

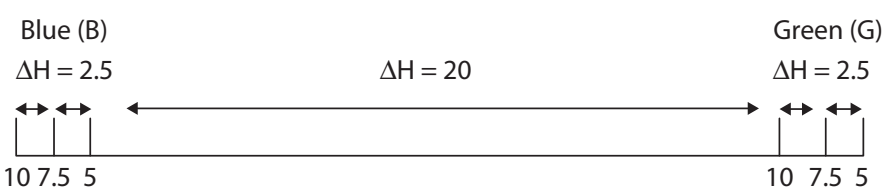

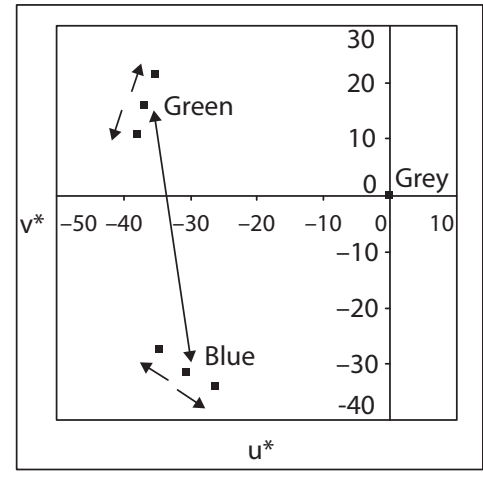

Location in $\mathrm{CIE} \mathrm{u}^{*}, \mathrm{~V}^{*}$

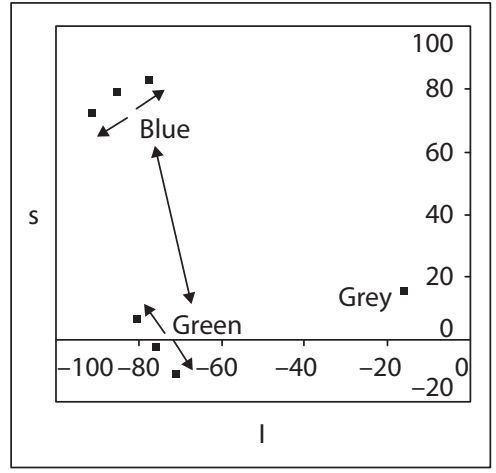

Location in DKL 1,s

Figure 1. Positions of training and test stimuli in colour space. The training colours were Munsell 7.5G and 7.5B (value/chroma 6/6). The top panel is a linear representation of a portion of Munsell hue space showing the 2.5 hue unit separation between the 'near' hues and the 15 hue unit separation between the closest green and blue hues experienced during testing. The left and right lower panels show these same colours represented in CIE and DKL colour spaces

In separate trials, stimuli were presented at two locations. These locations were centred $7^{\mathrm{o}}$ from fixation in the upper right and lower right quadrants of the visual field. The centre-to-centre separation of the two locations was 9.9 .

Stimuli were drawn from two sets of three Munsell colours - blues (5B, 7.5B, 10B), and greens $(5 \mathrm{G}, 7.5 \mathrm{G}, 10 \mathrm{G}$ ), at constant Chroma and Value of 6/6. See Figure 1 for the locations of the stimuli in CIE and DKL colour spaces.

\subsection{Design}

Observers were randomly allocated into one of four training conditions that varied the training stimulus location (top or bottom right display quadrant) and the training hue (7.5G 'Green' or 7.5B 'Blue'). Thus, the four training groups were designated top green, top blue, bottom green or bottom blue.

On day 1 and day 10, observers' discrimination thresholds for both sets of colour stimuli (green and blue) were measured at both locations (top and bottom) in order to assess their pre- and post-training thresholds. Between these measurements, on days 2 
to 9 , observers were trained just at their training location with their training hue. Thus, we were able to measure the effect of training on chromatic thresholds at the training hue/location and the extent of generalization of learning to another location, to other hues in the same colour category ('near' \pm 2.5 Munsell hue units relative to the training hue) and to hues in a different category ('far' 17.5, 20 or 22.5 Munsell hue unit difference from the training hue).

\subsection{Procedure}

The task was to discriminate whether the boundary between the two colours sloped to the left or right (see Figure 2).

To focus on 'low-level' perceptual analysis we selected a large orientation difference $\left(90^{\circ}\right)$, which produces threshold measures that are similar to those for detection (Thomas \& Gille 1979). The discrimination thresholds were measured using the ZEST algorithm (King-Smith, Grigsby, Vingrys, Benes \& Supowit 1994) ${ }^{1}$, which was set to

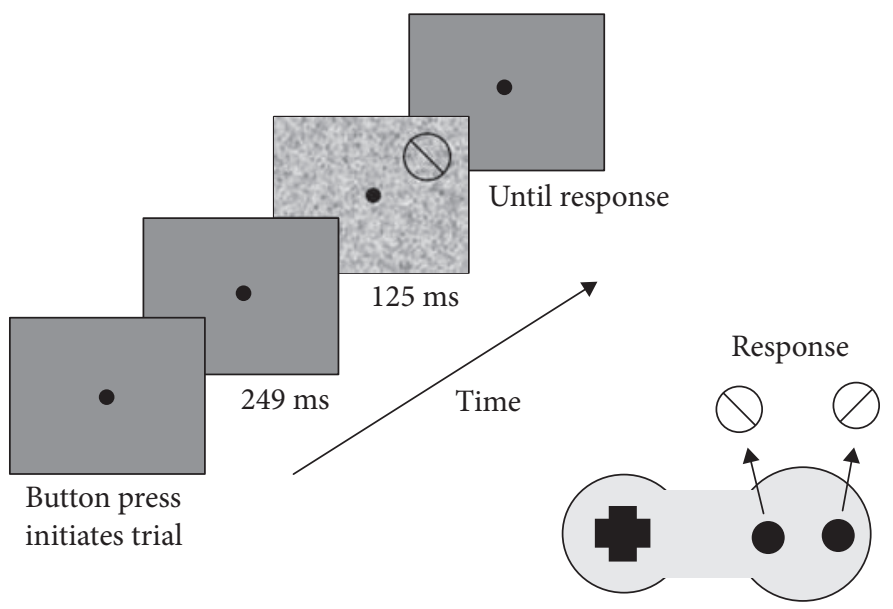

Figure 2. Schematic representation of the trial structure. The observer initiated each trial by pressing a button. After $249 \mathrm{msec}$ the stimulus was briefly presented (125 msec) such that the observer would not have time to move their eyes from fixation to the stimulus. The observer used the buttons on the gamepad to indicate whether the colour defined boundary was tilted left or right

1. The ZEST algorithm is a Bayesian adaptive threshold estimation procedure that continuously modifies an assumed a-priori probability density (pdf) function, which represents the probability that threshold is at each of a range of levels of stimulus intensity (or chromatic difference as used here), on the basis of the preceding response, and sets the difficulty of the next trial to be the mean of the current pdf function. In this way all of an observer's previous responses are taken into account in setting the difficulty of the next trial. 
converge on threshold at $82 \%$ correct discrimination. Three ZEST runs were randomly interleaved lasting 32 trials each and threshold was estimated as the average of the three runs. In this case the algorithm varied the size of the hue difference between the two halves of the circle around the current threshold measurement point along a linear line through colour space that also passed through the other 'near' points for that hue category.

The order of measuring the different thresholds for the various hue/location combinations on Day 1 was randomized for each participant and then held constant when the measurements were repeated on Day 10.

On days 2-9 participants completed six blocks of training (96 trials per block comprising three randomly interleaved ZEST runs of 32 trials each) on their training colour at their training location.

\section{Results}

Statistical analysis was conducted using Analysis of Variance (ANOVA) with Bonferroni corrected post-hoc tests. The results of the ANOVA analyses are reported in Tables 1 and 2, with p-values for the post-hoc tests shown in the text. Below we describe the statistically significant findings.

\subsection{Transfer across location}

We explored the effects of training on thresholds for the trained and untrained locations and on hues in the same region of colour-space (see Figure 3).

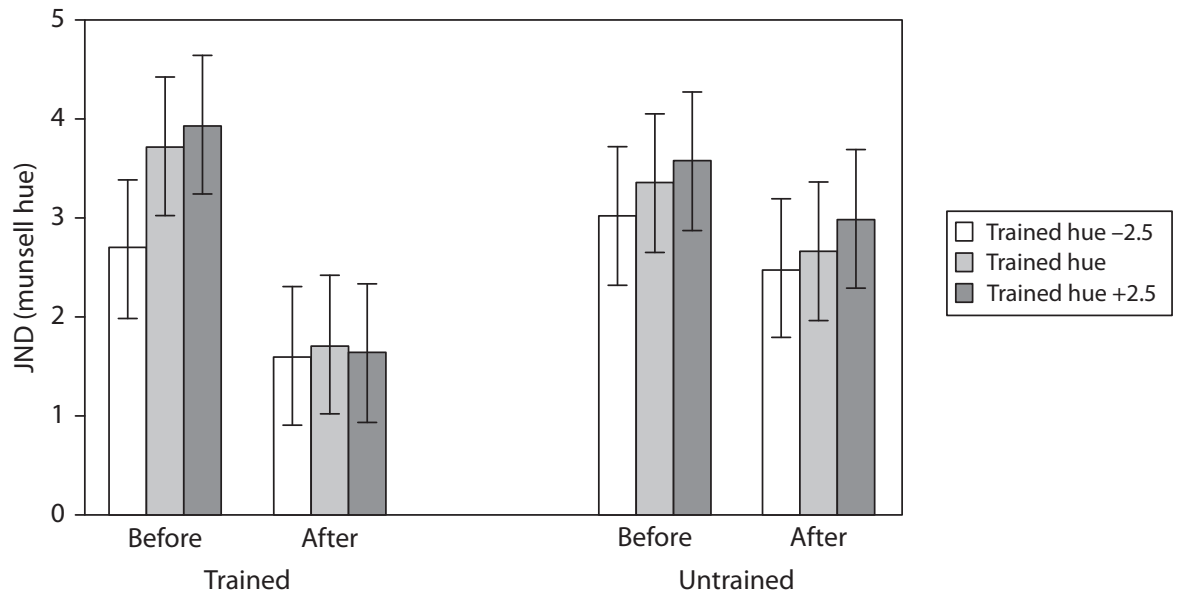

Figure 3. Graph to show threshold (in Munsell hue units) for discriminating the orientation of a chromatically defined edge before and after training. Separate bar charts are shown for trained and untrained retinal locations and separate bars are shown for the training hue and adjacent 'near' hues 
Table 1. Statistically significant results of three-way ANOVA to explore the effects of training on thresholds for the trained and untrained locations and on hues in the same region of colour-space (time (2) - pre-training, post-training; location (2) - trained location, untrained location; near hue (3) $--2.5,0$ and +2.5 Munsell hue steps relative to the training hue). The Greenhouse-Geisser correction to the degrees of freedom is made when the assumption of sphericity is violated (assessed by Mauchley's w)

\begin{tabular}{lccccc}
\hline Effect & $F$ & $d f$ & $p$ & $\begin{array}{c}\text { Partial } \\
\text { Eta2 }\end{array}$ & $\begin{array}{c}\text { Observed } \\
\text { power }\end{array}$ \\
\hline Time & 7.62 & 1,14 & $<0.05$ & 0.35 & 0.73 \\
Near Hue & 5.72 & $1.24,17.43$ & $<0.05$ & 0.29 & 0.83 \\
Time x Location & 7.72 & 1,14 & $<0.05$ & 0.36 & 0.73 \\
Time x Location x Near Hue & 3.35 & 2,28 & $<0.05$ & 0.19 & 0.56 \\
\hline
\end{tabular}

In general, thresholds decreased following training (main effect of time; see Table 1). In other words, participants become better at the task with practice.

Overall performance also differed between the 'near' hues (main effect of near hue; Table 1), with better performance on the -2.5 hue than the +2.5 hue $(p<0.05)$. Of particular interest, thresholds decreased significantly at the trained location $(p<0.0005)$ but not at the untrained location $(p=0.39)$ (interaction between time and location; Table 1).

Finally, for the trained but not untrained location, before training thresholds for the -2.5 'near' hue were significantly lower than for the 0 or +2.5 hues $(p$ 's $<0.05)$, which did not differ $(p=1.0)$. After training none of the thresholds differed significantly across the near hues ( $p$ 's $=1.0$ ) (interaction between time, location and near hue; Table 1).

\subsection{Transfer across colour}

We explored the effects of training on thresholds for the trained and untrained colours (blue or green) and on hues in the same region of colour-space (see Figure 4).

In general, thresholds decreased following training (main effect of time; Table 2). In other words participants become better at the task with practice.

Overall performance also differed between the 'near' hues (main effect of near hue; Table 2), with better performance on the -2.5 hue than the 0 hue $(p<0.05)$. Of particular interest, thresholds decreased significantly for the trained colour $(p<0.0005)$ but not for the untrained colour $(p=0.12)$ (interaction between time and colour; Table 2). 

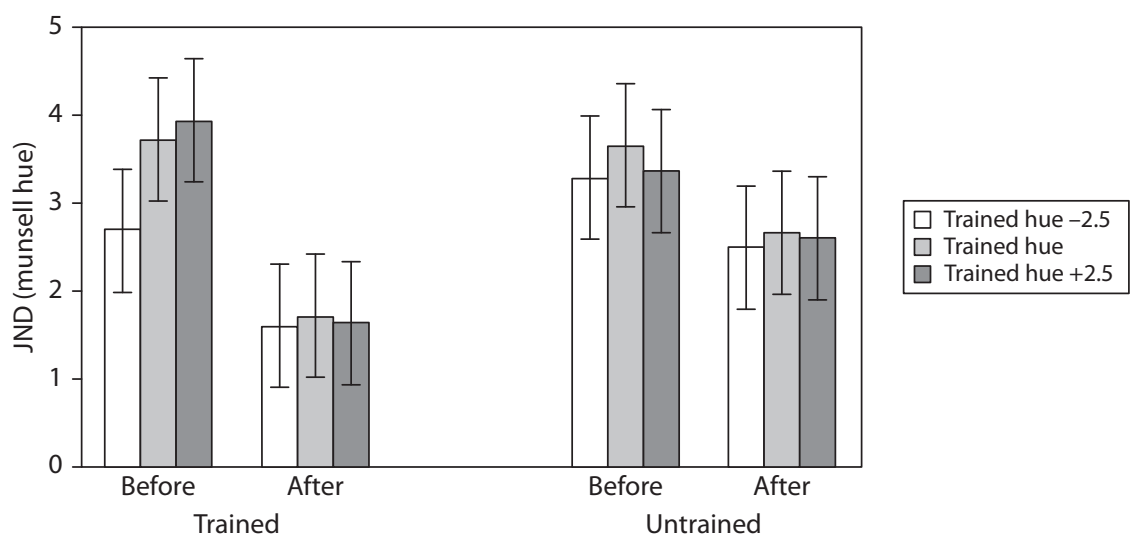

Figure 4. Graph to show threshold (in Munsell hue units) for discriminating the orientation of a chromatically defined edge before and after training. Separate bar charts are shown for trained and untrained hues and separate bars are shown for the trained/untrained hue and adjacent 'near' hues

Table 2. Statistically significant results of three-way ANOVA to explore the effects of training on thresholds for the trained and untrained colours and on hues in the same region of colour-space (time (2) - pre-training, post-training; colour (2) - trained colour, untrained colour; near hue (3) $--2.5,0$ and +2.5 Munsell hue steps relative to the training hue). The Greenhouse-Geisser correction to the degrees of freedom is made when the assumption of sphericity is violated (assessed by Mauchley's w)

\begin{tabular}{lccccc}
\hline Effect & F & df & p & $\begin{array}{c}\text { Partial } \\
\text { Eta2 }\end{array}$ & $\begin{array}{c}\text { Observed } \\
\text { power }\end{array}$ \\
\hline Time & 10.58 & 1,14 & $<0.01$ & 0.43 & 0.86 \\
Near Hue & 4.57 & 2,28 & $<0.05$ & 0.25 & 0.73 \\
Time x Colour & 4.51 & 1,14 & $=0.052$ & 0.24 & 0.51 \\
\hline
\end{tabular}

\section{Discussion}

Previous research has indicated that performance on a wide variety of visual tasks can improve with practice. In the present experiment we sought to explore whether similar performance improvements can be observed for chromatically based judgements and to probe the potential neural locus of these improvements.

We found that eight days of practice at making judgements about the orientation of a chromatically defined boundary improved discrimination performance. This performance improvement did not transfer to another retinal location that was 
approximately $10^{\circ}$ from the training location. At early stages of visual processing, such as $\mathrm{V} 1$, cell receptive fields at our stimulus eccentricity tend to be small $\left(0.5^{\circ}\right)$, whilst as we progress through the various stages of the visual processing hierarchy receptive field sizes tend to increase up to around $5.5^{\circ}$ in area V4 and to $20^{\circ}$ or more in anterior inferotemporal cortex (AIT) (Kastner, Weerd, Pinsk, Elizondo, Desimone, \& Ungerleider 2001; Smith, Singh, Williams \& Greenlee 2001). Thus, our finding of positional specificity to within at least $10^{\circ}$ is consistent with learning localized to relatively early stages of visual analysis that deal with the processing of chromatic stimulus properties. Future work could probe the limits of this specificity to retinal position to provide a finer-grained estimate of the likely neural locus of the learning.

Our observation that improvements in chromatic discrimination based judgements transfer within a hue category, but not to a different hue category, bears an intriguing resemblance to colour CP. Recall that, in colour CP, discrimination of colours that fall within a category is less acute than discrimination of colours that are placed in different categories. Consequently, we might expect that in the present case learning would transfer to similar 'near' hues in the same category because these hues are relatively indistinct from the training hue. However, a true test of whether transfer patterns across hue reflect the categorical structure of colour space would compare transfer to near hues within a category to equally different hues from a neighbouring category. This test must remain the subject of future experiments.

Initial processing of the visual scene is conducted, in part, by two separate but interacting streams concerned with achromatic and chromatic stimulus properties (Schiller \& Logothetis 1990). Previous perceptual learning research has largely focused on tasks that can be accomplished by attending to achromatic stimulus properties. Consequently, the extent to which judgements that require processing of chromatic stimulus properties can improve with training has remained substantially unknown. Here, the observation that adults' performance of a chromatically based discrimination can improve with practice extends the perceptual learning literature to the chromatic processing stream. This observation supports the expectation that plasticity and learning are a generic capacity of adult visual processing and are not restricted to specific aspects or streams of visual analysis.

Further, the observation of chromatic perceptual learning lends support to the possibility that colour categorical perception effects may arise as a result of learning during everyday experience. Specifically, the act of attending to a colour category boundary may drive a learning process that enhances discrimination of colours that straddle that boundary. Consequently, where a particular colour boundary occurs in one language but not another then we would expect speakers of the language that marks a given boundary to show relatively enhanced perception at that boundary, just as has been observed (e.g. Roberson et al. 2000). These language-driven effects need not involve permanent modifications to the early neural circuits processing colour. Instead they may be implemented dynamically as a chromatic task is performed, consistent with recent neuroimaging evidence (Siok et al. 2009). 


\section{Conclusions}

Chromatic discrimination judgements improve with practice and this improvement may reflect changes at early stages of visual analysis. This type of chromatic perceptual learning may partially underpin colour categorical perception and the differences in colour $\mathrm{CP}$ observed between the speakers of languages with differing colour vocabulary.

\section{Acknowledgements}

This work was funded by the ESRC (grant ref: RES-000-23-1011).

\section{References}

Drivonikou, Gilda V., Paul Kay, Terry Regier, Richard Ivry, Aubrey Gilbert, Anna Franklin \& Ian R. L. Davies. 2007. "Further evidence of Whorfian effects to the right visual field". Proceedings of the National Academy of Sciences 104.1097-1102.

Fahle, Manfred \& Tomaso Poggio. 2002. Perceptual Learning. Cambridge, Mass.: MIT Press.

— \& Shimon Edelman. 1993. "Long-term learning in vernier acuity: Effects of stimulus orientation, range and of feedback". Vision Research 33.397-412.

Fletcher, Robert. 1980. City colour vision test. Windsor: Keeler Ltd.

Franklin, Anna, Gilda V. Drivonikou, Ally Clifford, Paul Kay, Terry Regier \& Ian R. L. Davies 2008. "Lateralization of Categorical Perception of color changes with color term acquisition". Proceedings of the National Academy of Sciences, USA 47.18221-18225.

Furmanski, Chris S., Denis Schluppeck \& Steven A. Engel. 2004. "Learning strengthens the response of primary visual cortex to simple patterns". Current Biology 14.573-578.

Kastner, Sabine, Peter D. Weerd, Mark A. Pinsk, M. Idette Elizondo, Robert Desimone \& Leslie G. Ungerleider. 2001. "Modulation of sensory suppression: implications for receptive field sizes in human visual cortex". Journal of Neurophysiology 86.1398-1411.

King-Smith, P. Ewen, Scott S. Grigsby, Algis J. Vingrys, Susan C. Benes \& Aaron Supowit. 1994. "Efficient and unbiased modifications of the QUEST threshold method: Theory, simulations, experimental evaluation and practical implementation". Vision Research 34.885-912.

Mollon, John D. \& Marina V. Danilova. 1996. "Three remarks on perceptual learning”. Spatial Vision 10.51-58.

Özgen, Emre \& Ian R. L. Davies. 2002. "Acquisition of categorical color perception: A perceptual learning approach to the linguistic relativity hypothesis". Journal of Experimental Psychology: General 131.477-493.

Petrov, Alex A., Barbara A. Dosher \& Zhong-Lin Lu. 2005. "The dynamics of perceptual learning: An incremental reweighting model". Psychological Review 112.715-743.

Pourtois, Giles, Karsten S. Rauss, Patrik Vuilleumier \& Sophie Schwartz. 2008. "Effects of perceptual learning on primary visual cortex in humans". Vision Research 48.55-62. 
Roberson, Debi, Ian Davies \& Jules Davidoff. 2000. "Color categories are not universal: Replications and new evidence from a stone-age culture". Journal of Experimental Psychology: General 129.369-398.

Schwartz, Sophie, Pierre Maquet \& Chris Frith. 2002. "Neural correlates of perceptual learning: A functional MRI study of visual texture discrimination". Proceedings of the National Academy of Sciences, USA 99.17137-17142.

Schiller, Peter H. \& Nikos K. Logothetis. 1990. "The color-opponent and broad-band channels of the primate visual system". Trends in Neurosciences 13.392-398.

—, Nikos K. Logothetis \& E. R. Charles. 1991. "Parallel pathways in the visual system: Their role in perception at isoluminance". Neuropsychologia 29.433-441.

Shiu, Ling-Po \& Harold Pashler. 1992. "Improvement in line orientation discrimination is retinally local but dependent on cognitive set". Perception \& Psychophysics 52.582-588.

Siok, Wai T., Paul Kay, William S. Y. Wang, Alice H. D. Chan, Lin Chen, Kang-Kwong Luke \& Li H. Tan. 2009. "Language Regions of Brain Are Operative in Color Perception". Proceedings of the National Academy of Sciences, USA 106.8140-8145.

Smith, Andy T., Krish D. Singh, Adrian L. Williams \& Mark W. Greenlee. 2001. "Estimating receptive field size from fMRI data in human striate and extrastriate visual cortex". Cerebral Cortex 11.1182-1190.

Snowden, Robert J. 2002. "Visual attention to color: Parvocellular guidance of attentional resources?” Psychological Science 13.180-184.

Sowden, Paul T., David Rose \& Ian R. L. Davies. 2002. "Perceptual learning of luminance contrast detection: Specific for spatial frequency and retinal location but not orientation". Vision Research 42.1249-1258.

_ \& Philippe G. Schyns. 2006. "Channel surfing in the visual brain". Trends in Cognitive Sciences $10.538-545$.

Thomas, James P. \& Jennifer Gille. 1979. "Bandwidths of Orientation Channels in Human Vision". Journal of the Optical Society of America 69.652-660. 
